\title{
Crystal structure of the first orphan GPCR
}

\author{
Xi Lin, Fei Xu* \\ iHuman Institute, ShanghaiTech University \\ xufei@shanghaitech.edu.cn
}

The GPR52 receptor is a Class-A orphan G protein-coupled receptor (GPCR) whose endogenous ligand remains elusive. Highly expressed in the brain, it represents a promising therapeutic target for treating psychiatric disease and Huntington's disease. However, tool ligand and drug discovery have been largely hampered by a lack of structural information due largely to the low homology $(<20 \%)$ of GPR52 to any known GPCR structure. We reported three high resolution human GPR52 structures with and without a bound ligand. According to the structures, we observed a unique configuration of extracellular loop 2 (ECL2) that occupies the orthosteric pocket, a novel side pocket and a special winding mode for Transmembrane helix 5 (TM5). Mutagenesis and functional assay suggested the self-activation by ECL2. These findings provide unprecedented insights into the structural basis of GPR52 ligand recognition that will be valuable for GPR52 deorphanization and will guide the design of diverse ligands with distinct pharmacological properties that have not yet been possible.

Keywords: crystallography, orphan receptor, ligand binding mode 